\title{
Measurement of the cosmological distance scale using X-ray and Sunyaev-Zel'dovich effect observations of galaxy clusters
}

\author{
Massimiliano Bonamente, ${ }^{1}$ John Carlstrom, ${ }^{2}$ Eric Leitch, ${ }^{3}$ \\ Marshall Joy, ${ }^{4}$ Daniel Marrone, ${ }^{5}$ Adam Mantz, ${ }^{2}$ Stephen Muchovej, ${ }^{3}$ \\ Thomas Plagge, ${ }^{2}$ and Erik Reese ${ }^{6}$ \\ ${ }^{1}$ University of Alabama in Huntsville, USA \\ email: bonamem@uah.edu \\ ${ }^{2}$ University of Chicago, USA; ${ }^{3}$ California Institute of Technology and Owens Valley Radio \\ Observatory, USA $;{ }^{4}$ NASA Marshall Space Flight Center, USA; ${ }^{5}$ University of Arizona, USA; \\ ${ }^{6}$ University of Pennsylvania, USA
}

\begin{abstract}
X-ray and Sunyaev-Zeldovich effect (SZE) observations of galaxy clusters can be used to measure their distances independently of the cosmic distance ladder. We have determined the distance to 38 clusters of galaxies in the redshift range $0.14 \leqslant z \leqslant 0.89$ using X-ray data from the Chandra X-ray Observatory and SZE data from the Owens Valley Radio Observatory and the Berkeley-Illinois-Maryland Association interferometric arrays. We measure a Hubble constant of $\mathrm{H}_{0}=76.9_{-3.4}^{+3.9}{ }_{-8.0}^{+10.0} \mathrm{~km} \mathrm{~s}^{-1} \mathrm{Mpc}^{-1}$ (statistical followed by systematic uncertainties at $68 \%$ confidence) for an $\Omega_{\mathrm{M}}=0.3, \Omega_{\Lambda}=0.7$ cosmology. Our determination of the Hubble parameter in the distant Universe agrees with measurements from the Hubble Space Telescope Key Project that probed the nearby Universe.
\end{abstract}

Keywords. galaxies: clusters: general, cosmic microwave background, cosmology: observations, distance scale

\section{X-ray and Sunyaev-Zel'dovich effect observations of galaxy clusters}

Galaxy clusters are the largest gravitationally collapsed structures in the Universe, with a hot diffuse plasma $\left(T_{\mathrm{e}} \sim 10^{7}-10^{8} \mathrm{~K}\right)$ that fills the intergalactic space and is a strong emitter of X-ray radiation. Cosmic microwave background (CMB) photons passing through this hot intracluster medium have a $\sim 1 \%$ chance of inverse-Compton scattering off the plasma's energetic electrons, causing a small $(\sim 1 \mathrm{mK})$ distortion in the CMB spectrum, known as the Sunyaev-Zel'dovich effect (SZE; Sunyaev \& Zel'dovich 1970, 1972; for reviews, see Birkinshaw 1999; Carlstrom et al. 2002). The SZE causes a change in the temperature of the CMB as a function of the integrated pressure,

$$
\Delta T \propto \int n_{e} T_{\mathrm{e}} \mathrm{d} \ell
$$

where $n_{e}$ and $T_{\mathrm{e}}$ are the electron number density and temperature of the hot gas, and the integration is along the line of sight. The X-ray emission scales as

$$
S_{X} \propto \int n_{e}^{2} \Lambda_{e e} \mathrm{~d} \ell
$$

where $\Lambda_{e e}$ is the X-ray cooling function. 

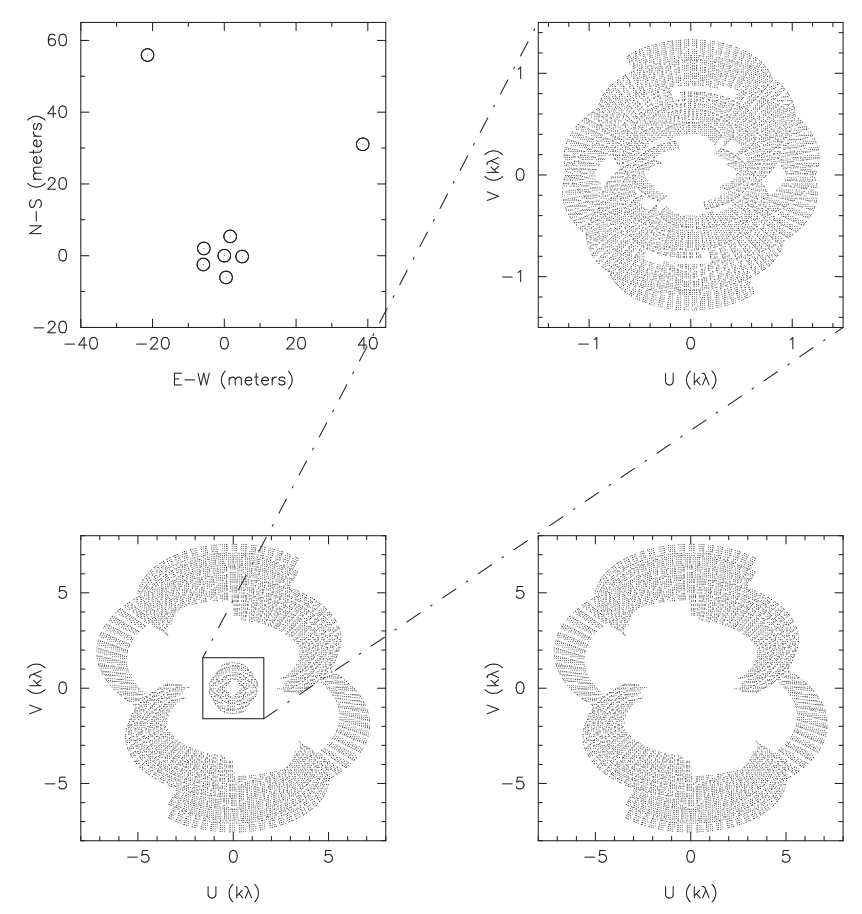


Figure 1. (top left) SZA telescope locations. (bottom left) Resulting Fourier-space $(u-v)$ coverage for a single track (all baselines). (top middle) $u-v$ coverage for the short baselines only $(0-2 k \lambda)$. (bottom middle) $u-v$ coverage for the long baselines only $(2-8 k \lambda)$. (top right) Resulting point-spread function (synthesized beam), short baselines. (bottom right) Synthesized beam, long baselines. (Reproduced with permission from fig. 1 of Muchovej et al. 2007, ApJ, 663, 710.)

The different dependences on density, along with a model of the cluster gas, enable a direct distance determination to a given galaxy cluster through the combined analysis of X-ray and SZE radio data (e.g., Reese et al. 2002; Bonamente et al. 2006). This method is independent of the extragalactic distance ladder, and therefore provides an independent estimate of the Hubble constant.

\section{Sunyaev-Zel'dovich effect observations}

In Bonamente et al. (2006) we reported SZE observations at $30 \mathrm{GHz}$ with the Owens Valley Radio Observatory (OVRO) and the Berkeley-Illinois-Maryland Array (BIMA) interferometric arrays for a sample of 38 clusters. Interferometric observations of clusters are illustrated in Fig. 1, showing the layout and observing methods of the SunyaevZel'dovich Array (SZA), an eight-element interferometer that we used for a cluster survey and additional pointed observations (e.g., Muchovej et al. 2007; Culverhouse et al. 2010; Bonamente et al. 2012). The presence of two long-baseline antennas results in the ability to detect and remove point sources present in the field of view. The SZA, OVRO, and BIMA antennas are now part of the Combined Array for Millimiter Astronomy (CARMA), a hybrid interferometric array with 30 and $90 \mathrm{GHz}$ receivers, capable of $10^{\prime \prime}$ angular resolution, for the detection of the SZE.

The SZE can also be observed with single-dish imaging methods. Current-generation single-dish radio observatories include the Planck satellite, the South Pole Telescope $(S P T)$ and the Atacama Cosmology Telescope $(A C T)$. These observatories have provided a wealth of cluster observations, including large-area surveys that can be used for 


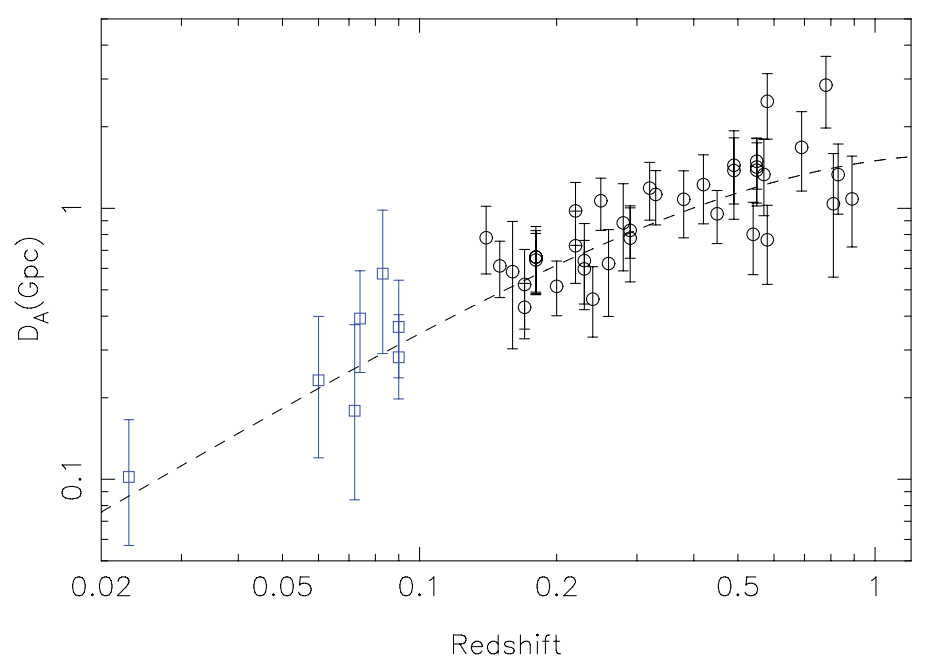

Figure 2. Angular-diameter distances of our 38 sample clusters (open circles). The error bars are the total statistical uncertainties, obtained by combining the X-ray and SZE data modeling uncertainties and additional sources of random error. The dashed line is the angular-diameter curve for the best-fitting Hubble constant $\mathrm{H}_{0}=76.9 \mathrm{~km} \mathrm{~s}^{-1} \mathrm{Mpc}^{-1}$ and $\Omega_{\mathrm{M}}=0.3, \Omega_{\Lambda}=0.7$. The open squares are from the low-redshift sample of Mason et al. (2001); they are not included in the fit. (Reproduced with permission from fig. 3 of Bonamente et al. 2006, ApJ, 647, 32.)

additional cosmological applications such as the measurement of dark-energy properties (e.g., Hincks et al. 2010; Staniszewski et al. 2010; Plagge et al. 2010; Ade et al. 2011).

\section{Measurement of the Hubble constant}

Using the 38 clusters observed with OVRO and BIMA, we obtained the Hubble diagram of Fig. 2. The angular-diameter distance, $D_{A}$, is a function of the cluster's redshift, $z$, the mass density, $\Omega_{\mathrm{M}}$, the dark-energy density, $\Omega_{\Lambda}$, and the Hubble constant, $\mathrm{H}_{0}$, which provides the overall normalization:

$$
D_{A}(z)=\frac{1}{\mathrm{H}_{0}} \frac{c}{\left|\Omega_{k}\right|^{1 / 2}(1+z)} \operatorname{sinn}\left[\left|\Omega_{k}\right|^{1 / 2} \int_{0}^{z}\left[(1+\zeta)^{2}\left(1+\Omega_{\mathrm{M}} \zeta\right)-\zeta(2+\zeta) \Omega_{\Lambda}\right]^{-1 / 2} \mathrm{~d} \zeta\right],
$$

where the function $\operatorname{sinn}(x)$ is defined as $\sinh (x)$ for $\Omega_{k}>0, \operatorname{sinn}(x)=x$ for $\Omega_{k}=0$, and $\operatorname{sinn}(x)=\sin (x)$ for $\Omega_{k}<0$, while $\Omega_{k}=1-\Omega_{\mathrm{M}}-\Omega_{\Lambda}$ (Carroll et al. 1992).

For an $\Omega_{\mathrm{M}}=0.3, \Omega_{\Lambda}=0.7$ cosmology, we obtain a best-fitting value of the Hubble constant of $\mathrm{H}_{0}=76.9_{-3.4}^{+3.9}{ }_{-8.0}^{+10.0} \mathrm{~km} \mathrm{~s}^{-1} \mathrm{Mpc}^{-1}$ (statistical followed by systematic uncertainty at $68 \%$ confidence). This fit is obtained with a model of the cluster density and temperature that takes into account a radial profile for the gas temperature. We also analyzed the data using an isothermal $\beta$ model that does not invoke the hydrostaticequilibrium assumption, and find $\mathrm{H}_{0}=73.7_{-3.8}^{+4.6}{ }_{-7.6}^{+9.5} \mathrm{~km} \mathrm{~s}^{-1} \mathrm{Mpc}^{-1}$. To avoid effects from cool cores in clusters, we repeated this analysis excluding the central $100 \mathrm{kpc}$ from the X-ray data, and find $\mathrm{H}_{0}=77.6_{-4.3}^{+4.8}{ }_{-8.2}^{+10.1} \mathrm{~km} \mathrm{~s}^{-1} \mathrm{Mpc}^{-1}$. This measurement of the Hubble constant for a dark-energy-dominated concordance cosmology (e.g., Komatsu et al. 2011) is in agreement with the Hubble Space Telescope Key project measurements (e.g., Freedman et al. 2001, 2012). 

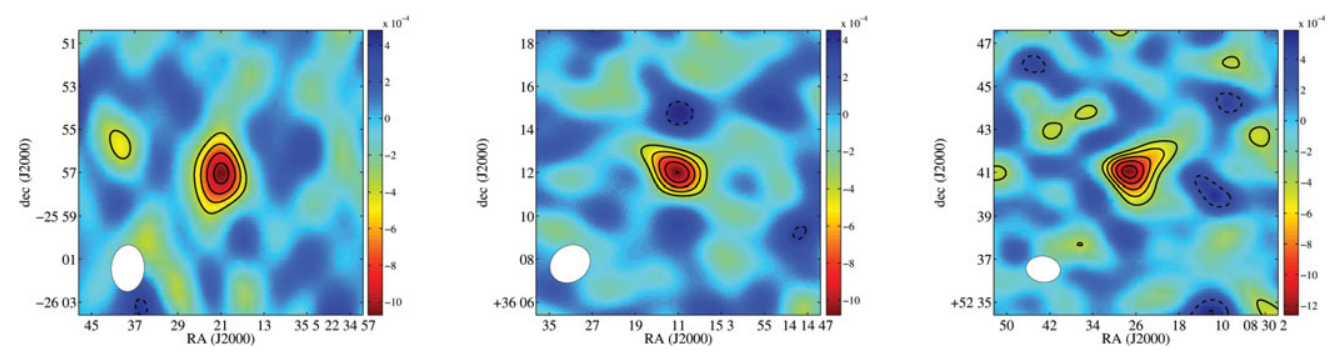

Figure 3. CLEANed SZA short-baseline $(<2 k \lambda)$ images of three clusters with significant SZE detections. From left to right, XMMU J2235-2557, Cl J1415.1+3612, and 2XMM $\mathrm{J} 083026.2+524133$, with the color scale given in $\mathrm{Jy}_{\text {beam }}^{-1}$. The contours start at $2 \sigma$ and are spaced at unit intervals of the map's rms $\sigma$. The white ellipse represents the half-power point of the elliptical 2D Gaussian function that approximates the main lobe of the synthesized beam. Radio sources have been removed for display purposes. (Reproduced with permission from fig. 1 of Culverhouse et al. 2010, ApJ, 723, L80.)

\section{Prospects for future measurements of the Hubble constant with galaxy clusters}

X-ray observations of clusters with current-generation X-ray satellites (Chandra, XMMNewton, and Suzaku) and radio interferometers (such as the SZA and CARMA) can be further used to measure cluster distances and the Hubble constant. Our group is analyzing a sample of approximately 100 clusters observed with CARMA/SZA, for which we have X-ray data available (primarily from Chandra). In Fig. 3 we show a sample of three clusters at redshift $z \geqslant 1$ observed by the $S Z A$. The use of high-redshift clusters provides a unique, direct probe of the expansion of the Universe at distances $\leqslant 1 \mathrm{Gpc}$. The resolution and angular sensitivity of the $S Z A$ represent a significant improvement over the OVRO and BIMA data used by Bonamente et al. (2006). SZA observations of 25 clusters from the Allen et al. (2008) sample resulted in the direct measurement of the gas pressure from SZE data alone, as shown in Fig. 4 (Bonamente et al. 2012). One of the goals of further analyses of our CARMA/SZA cluster observations is to provide an improved measurement of the Hubble constant with reduced uncertainties.

Upcoming facilities include an upgrade of the CARMA receivers to achieve finer angular resolution and a wider passband, and new X-ray satellites such as ASTRO-H and e-Rosita. Cluster data that will become available in the next few years will therefore contribute to the reduction in both the statistical and systematic error in the available measurements of the Hubble constant with clusters. The samples of clusters that will be available for this study will increase by approximately one order of magnitude, thus reducing the statistical errors. The new data will also enable further studies of such sources of systematic uncertainties as clumping in the cluster gas and the presence of non-thermal pressure support.

\section{Conclusions}

X-ray and SZE observations of galaxy clusters provide a unique method to measure distances to distant galaxy clusters, and therefore to measure the Hubble constant. Available measurements for large cluster samples include Reese et al. (2002) and Bonamente et al. (2006), the latter for a sample of 38 clusters at redshifts $z=0.14-0.89$, for which we measured a Hubble constant of $\mathrm{H}_{0}=76.9_{-3.4}^{+3.9}{ }_{-8.0}^{+10.0} \mathrm{~km} \mathrm{~s}^{-1} \mathrm{Mpc}^{-1}$ (statistical followed by systematic uncertainty at $68 \%$ confidence). Several current-generation and upcoming 


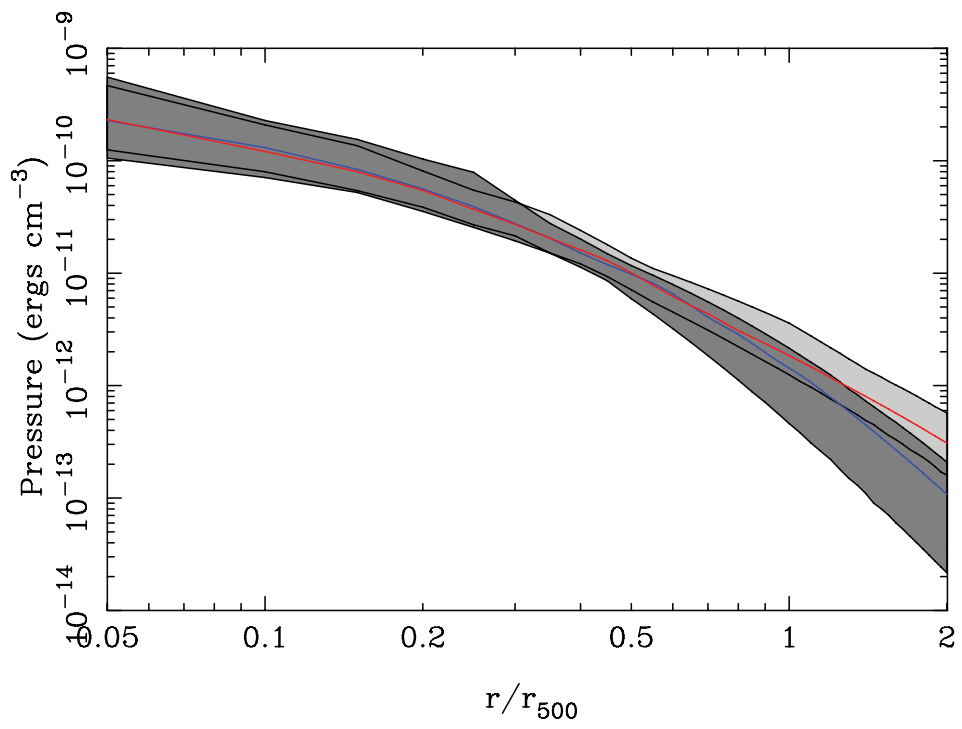

Figure 4. Average pressure profiles from SZ fits to the Bulbul et al. (2010) model (dark grey area, blue line), and to the Arnaud et al. (2010) model (light grey area, red line). The lines are the medians of the 25 best-fitting distributions, and the error bands are the $68 \%$ confidence levels. (Reproduced from Bonamente et al. 2012.)

X-ray and SZE facilities will provide new measurements of the Hubble constant with significant reduction in both statistical and systematic uncertainties.

\section{References}

Ade, P. A. R., Aghanim, N., \& Arnaud, M., 2011, A\&A, 536, A8

Allen, S. W., Rapetti, D. A., Schmidt, R. W., Ebeling, H., Morris, R. G., \& Fabian, A. C. 2008, $M N R A S, 383,879$

Arnaud, M., Pratt, G. W., Piffaretti, R., Böhringer, H., Croston, J. H., \& Pointecouteau, E. 2010, A\& A, 517, A92

Birkinshaw, M. 1999, Phys. Rep., 310, 97

Bonamente, M., Hasler, N., Bulbul, E., et al. 2012, New J. Phys., 14, 025010

Bonamente, M., Joy, M. K., LaRoque, S. J., Carlstrom, J. E., Reese, E. D., \& Dawson, K. S. 2006, ApJ, 647, 25

Bulbul, G. E., Hasler, N., Bonamente, M., \& Joy, M. 2010, ApJ, 720, 1038

Carlstrom, J. E., Holder, G. P., \& Reese, E. D. 2002, ARA\&AA, 40, 643

Carroll, S. M., Press, W. H., \& Turner, E. L. 1992, ARAEBA, 30, 499

Culverhouse, T. L., Bonamente, M., Bulbul, E., et al. 2010, ApJ, 723, L78

Freedman, W. L., Madore, B. F., Gibson, B. K., et al. 2012, ApJ, 758, 24

Hincks, A. D., Acquaviva, V., Ade, P., et al. 2010, ApJS, 191, 423

Komatsu, E., Smith, K. M., Dunkley, J., et al. 2011, ApJS, 192, 18

Mason, B. S., Myers, S. T., \& Readhead, A. C. S. 2001, ApJ, 555, L11

Muchovej, S., Mroczkowski, T., Carlstrom, J. E., et al. 2007, ApJ, 663, 708

Plagge, T., Benson, B. A., Ade, P. A. R., et al. 2010, ApJ, 716, 1118

Staniszewski, Z., Ade, P. A. R., Aird, K. A., et al. 2009, ApJ, 701, 32

Sunyaev, R. A. \& Zel'dovich, Y. B. 1970, Comm. Astrophys. Space Phys., 2, 66

Sunyaev, R. A. \& Zel'dovich, Y. B. 1972, Comm. Astrophys. Space Phys., 4, 173 